\title{
Consecuencias psíquicas en supervivientes permanentes de cáncer de mama. Curadas, aunque no libres
}

\section{Psychological Consequences in Long-Term Breast Cancer Survivors: Recovered but not Free}

\author{
María-José Fernández-Guerrero y Beatriz Palacios-Vicario \\ Universidad Pontificia de Salamanca, España
}

\begin{abstract}
Resumen: El presente artículo de reflexión teórica se centra en analizar las necesidades de un grupo de mujeres que han sufrido un cáncer de mama, denominadas "supervivientes permanentes" o "a largo plazo" en las que, transcurridos más de cinco años tras el tratamiento primario, continúan con secuelas tanto físicas como psíquicas. Para ello se realizó un análisis exploratorio de la literatura existente en bases de datos del ámbito de la salud, en un periodo temporal de 2015 a 2019. Se analizan los conceptos de "superviviente" y "superviviente a largo plazo", los síntomas psíquicos y físicos que se mantienen más allá de los 5 años post-tratamiento y la posible existencia de un trastorno de estrés postraumático por cáncer (más allá del miedo a la recidiva). Finalmente, se plantea el debate acerca del concepto de superviviente como identidad adjudicada, en la cual no todas las mujeres se reconocen.

Palabras clave:_supervivencia a largo plazo, cáncer de mama, psicooncología, trastorno de estrés postraumático por cáncer, identidad, efectos a largo plazo.
\end{abstract}

\begin{abstract}
This article is a theoretical reflection focusing on the analysis of the needs of a group of women who have suffered from breast cancer, called "long-term survivors" in whom both physical and psychological sequelae persist more than five years after primary treatment. To do this, an exploratory analysis of the existing literature was carried out in da-
\end{abstract}

\footnotetext{
María-José Fernández-Guerrero (iD https://orcid.org/0000-0002-2581-8437 es psicóloga, profesora en la Facultad de Psicología de la Universidad Pontificia de Salamanca, España.

Beatriz Palacios-Vicario (iD https://orcid.org/0000-0002-3559-9995 es psicopedagoga, profesora en la Facultad de Psicología de la Universidad Pontificia de Salamanca, España.

Para citar este artículo: Fernández-Guerrero, M. J. y Palacios-Vicario, B. (2021). Consecuencias psíquicas en supervivientes permanentes de cáncer de mama. Curadas, aunque no libres. Clínica Contemporánea, 12(3), Artículo e24. https://doi.org/10.5093/cc2021a17

La correspondencia sobre este artículo debe enviarse a las autoras al email mjfernandezgu@upsa.es (María-José Fernández-Guerrero) o bpalaciosvi@upsa.es (Beatriz Palacios-Vicario).
}

(cc) BY-NC-ND Este es un artículo Open Access bajo la licencia CC BY-NC-ND. 
tabases in the health field, over a period from 2015 to 2019. The concepts of "survivor" and "long-term survivor", the psychological and physical symptoms that persist for more than 5 years post-treatment, and the possible existence of cancer-related post-traumatic stress disorder (beyond the fear of relapse) are analyzed. Finally, a debate is raised about the concept of survivor as an adjudicated identity not actually shared by all women it seeks to define.

Keywords: long-term survivorship, breast cancer, psycho-oncology, cancer-related post-traumatic stress disorder, identity, long-term symptoms.

Los avances en la detección precoz, el diagnóstico y los tratamientos están favoreciendo que el cáncer en general, y el de mama en particular, haya dejado de ser una enfermedad con un desenlace fatal para constituir un cuadro con unos índices de recuperación en torno al 80-85\% (Asociación Española Contra el Cáncer [AECC], 2019; Sociedad Española de Oncología Médica [SEOM], 2017). A esta situación favorable se añade la reducción de los efectos secundarios de los tratamientos, una mejoría de la calidad de vida, y un progresivo incremento de la supervivencia, tanto en cantidad de personas como en años de la misma. El número de nuevos casos de cáncer va aumentando, pero la curación y la persistencia en fases libres de la enfermedad también.

Este hecho, afortunado y esperanzador, lleva consigo la aparición de un nuevo grupo de población que, en palabras de Ferro y Borràs (2011), empieza a constituir una "bola de nieve" en los servicios sanitarios: personas supervivientes de cáncer que han pasado la etapa de detección y diagnóstico, han sido sometidas a tratamiento (cirugía, quimioterapia, radioterapia, tratamiento hormonal o inmunoterapia) y ya están libres de la enfermedad. Es decir, se ha seguido el protocolo de prevenir, diagnosticar, tratar la fase activa y realizar un seguimiento durante unos años tras la remisión completa. Es en este punto donde se inserta la psicooncología como una necesidad más en el duro proceso de enfrentarse a un diagnóstico y a los procedimientos terapéuticos que de él se deriven.

Pero una vez que la paciente es dada de alta, y cuando ya se le puede considerar curada, las secuelas siguen presentes. El golpe emocional que ha supuesto enfrentarse al sufrimiento físico o al cuestionamiento sobre la propia finitud pasa factura. Y, yendo más allá, permanecen aspectos que le recordarán siempre que ha tenido (ha sufrido) cáncer de mama $(\mathrm{CM})$.

El objetivo del presente artículo se dirige a poner de manifiesto la existencia de un grupo de personas cada vez mayor que, habiendo superado un CM, sigue experimentando necesidades, efectos secundarios y síntomas psíquicos. En este sentido, nuestro foco de interés se centra en mujeres supervivientes a largo plazo de cáncer de mama: puesto que se pone el límite de la atención sanitaria en los cinco años tras finalizar el tratamiento primario, planteamos la existencia de sufrimiento psíquico que, en mayor o menor medida, sigue incidiendo en la fase de supuesto retorno a la normalidad (o, quizá, a una nueva normalidad), cuando ya están sanas y alejadas de tratamientos médicos. En resumen, y parafraseando a Roales-Nieto (2016), queremos indagar en la forma en la que "sobreviven a la supervivencia", habida cuenta de la persistencia de efectos tardíos y crónicos, tanto psíquicos como físicos.

Este grupo de personas a día de hoy no es una población diana en los protocolos de atención, en la mayoría de los programas formativos en Psicooncología ni, por ende, en la investigación nacional e internacional, si bien desde hace algunos años algo está empezando a cambiar. Una de las primeras llamadas de atención hacia la supervivencia a largo plazo como una etapa susceptible de ser incorporada en los protocolos, fue realizada en 2006 por Hewitt et al. en el marco de la American Society of Clinical Oncology publicaron un libro con el esclarecedor título "From cancer patient to cancer survivor: lost in transition", perdidos en la transición. Desde 2007 se publica una revista con el título Journal of Cancer Survivors (si bien adscrita a las áreas de Enfermería y Medicina); en Alemania se ha realizado el estudio CAESAR+ (Cancer SurvivorshipA multi-regional population-based study) para evaluar la calidad de vida de pacientes curados que fueron diagnosticados entre 1994 y 2004. Desde 1988 en Estados Unidos se celebra el primer domingo de junio el 
Día Nacional del Superviviente, estela que, en 2012, siguió nuestro país a instancias de la GEPAC (Grupo Español de Pacientes con Cáncer) fijándose su celebración en el día 3 de junio.

Aparte de la visibilidad que supone su "día internacional", en España también se van abriendo caminos a nivel asistencial. En 2017, el Hospital de Fuenlabrada (Madrid), en colaboración con la Fundación Sandra Ibarra, creó la Escuela de Supervivientes, un proyecto en el que se pretende dar a conocer el cáncer desde el punto de vista de las personas que lo han padecido, así como sus problemas y necesidades. Este proyecto se ha visto complementado con la creación de la primera Unidad del Superviviente de Cáncer en marzo de 2019. También es pionera la Unidad de Largos Supervivientes puesta en marcha en el Hospital de Salamanca en 2013, por el Dr. Juan Jesús Cruz y su equipo multidisciplinar, donde abordan las necesidades de pacientes que no han sufrido remisiones en 10 años. En 2012, la SEOM publicó su Manifiesto sobre la Atención a los Largos Supervivientes de Cáncer, reeditándolo en 2018 con el Plan Integral de Atención a Largos Supervivientes, donde estructuran una atención coordinada con Atención Primaria e implicando a diferentes profesionales, psicólogos clínicos y psicooncólogos entre ellos. En diciembre de 2019, el Institute for Healthcare Management de ESADE y los laboratorios AstraZeneca han puesto en marcha el "Proyecto ASISTO. El futuro ASISTencial Oncológico en largos supervivientes", con el fin de obtener información cuantitativa y cualitativa para modificar el sistema asistencial de cara a la satisfacción de necesidades de este amplio grupo de población.

\section{Planteamiento del problema}

Los problemas no siempre terminan cuando se supera el cáncer. Aunque una buena parte de los supervivientes a largo plazo viven el resto de sus vidas con una práctica ausencia de secuelas, los estudios y la experiencia clínica muestran cómo después de varios años de acabado el tratamiento primario siguen surgiendo síntomas psíquicos y físicos, unos secundarios al cáncer inicial y otros como consecuencia de la exposición a los tratamientos (Aaronson et al., 2014; Galindo et al. 2014; Harrington et al. 2010), lo que nos hace pensar en la necesidad de una atención especializada en esta etapa libre de enfermedad.

En una amplia revisión sistemática realizada en 2010, Harrington et al. repasaron la evidencia de síntomas en supervivientes a largo plazo de distintos tipos de cáncer (con el esclarecedor título de It's not over when it's over -no se acaba cuando acaba). Respecto al CM en concreto reseñaron que los síntomas más reportados en las distintas investigaciones fueron el cansancio, los trastornos del sueño, los síntomas depresivos y ansiosos, problemas cognitivos, dolores, limitaciones funcionales y alteraciones sexuales. De ellos, el cansancio, los síntomas depresivos y ansiosos, los síntomas dolorosos o neuropatías y las disfunciones sexuales seguían presentes después de los 5 años postratamiento.

Asimismo, García-Vivar y McQueen (2005) y García-Vivar (2012), en sus revisiones de la literatura, describen la existencia de problemas referidos a la imagen corporal, síntomas menopáusicos prolongados y de fertilidad, preocupaciones laborales y temor a la recurrencia, junto a las consecuencias psíquicas de los efectos físicos derivados del tratamiento, como mastectomía, linfedema o neuropatías periféricas (sobre todo en manos, pies y espalda), todo lo cual repercute de forma significativa en la calidad de vida de las supervivientes y en el mantenimiento del malestar psíquico. Así, como señala García-Vivar (2012) "las mujeres que en su día fueron diagnosticadas de CM pueden tener necesidades durante esta etapa de larga supervivencia, aunque parece que no siempre los sistemas sanitarios cubren estas necesidades" (p.289). De hecho, como se verá más adelante, también van decreciendo las investigaciones sobre este grupo de personas conforme aumentan los años de curación: aun hallando un interés importante en la supervivencia inmediata, las referencias a la supervivencia a largo plazo (más de 5 años) son escasas. En la revisión realizada en 2010 por Harrington et al. antes citada, se hallaron 21 estudios (en una horquilla de 2000 a 2008) donde se exploraban los síntomas que persistían tras más de 5 años. De estos estudios, ninguno incidía específicamente en el CM. España tampoco destaca por el interés investigador en este campo, ya que, salvando los estudios de García-Vivar citados anteriormente, no se han encontrado documentos publicados en nuestro país que se focalicen en los posibles síntomas psíquicos de la etapa libre de enfermedad > 5 años (de curación o remisión completa). 
Para comprobar estas afirmaciones y con carácter exploratorio, no sistemático, realizamos una búsqueda en las principales bases de datos: Psicodoc, PsycInfo, Medline (PubMed) y WOS (Clarivate Analytics) con los términos "long term surviv*”+"breast cancer" en un lapso temporal entre 2015 y 2019.

De los 96 documentos hallados inicialmente y tras la eliminación de las repeticiones, se obtuvieron 84 artículos, los cuales fueron sometidos a criterios de exclusión e inclusión (ver fig. 1). Así, tras eliminar aquellos cuyo estudio abarcara un periodo inferior a los 5 años postratamiento, que no versaran sobre CM o cuyo ámbito de trabajo no se centrara en aspectos psicopatológicos, se obtuvieron 15 resultados, de los cuales 8 realizaban una comparativa con otros tipos de cáncer y solo 7 abordaban consecuencias psíquicas en CM exclusivamente.

Para la determinación del estado de la investigación en nuestro país se utilizó la base de datos Psicodoc con los mismos términos de búsqueda y su equivalente en español, no hallando registros que satisficieran los criterios. Aunque este resultado parece indicar que puede existir una laguna sobre esta cuestión, se han localizado dos referencias en Pubmed de autoría española y con muestras procedentes del Área de Salud de la Costa del Sol y del Complejo Hospitalario de Granada, respectivamente, si bien publicadas en inglés en la revista $\mathrm{Su}$ pport Care Cancer (con sede en Alemania) (Álvarez-Salvago et al. 2018; Padilla-Ruiz et al. 2019).

Figura 1. Resultados de la búsqueda exploratoria

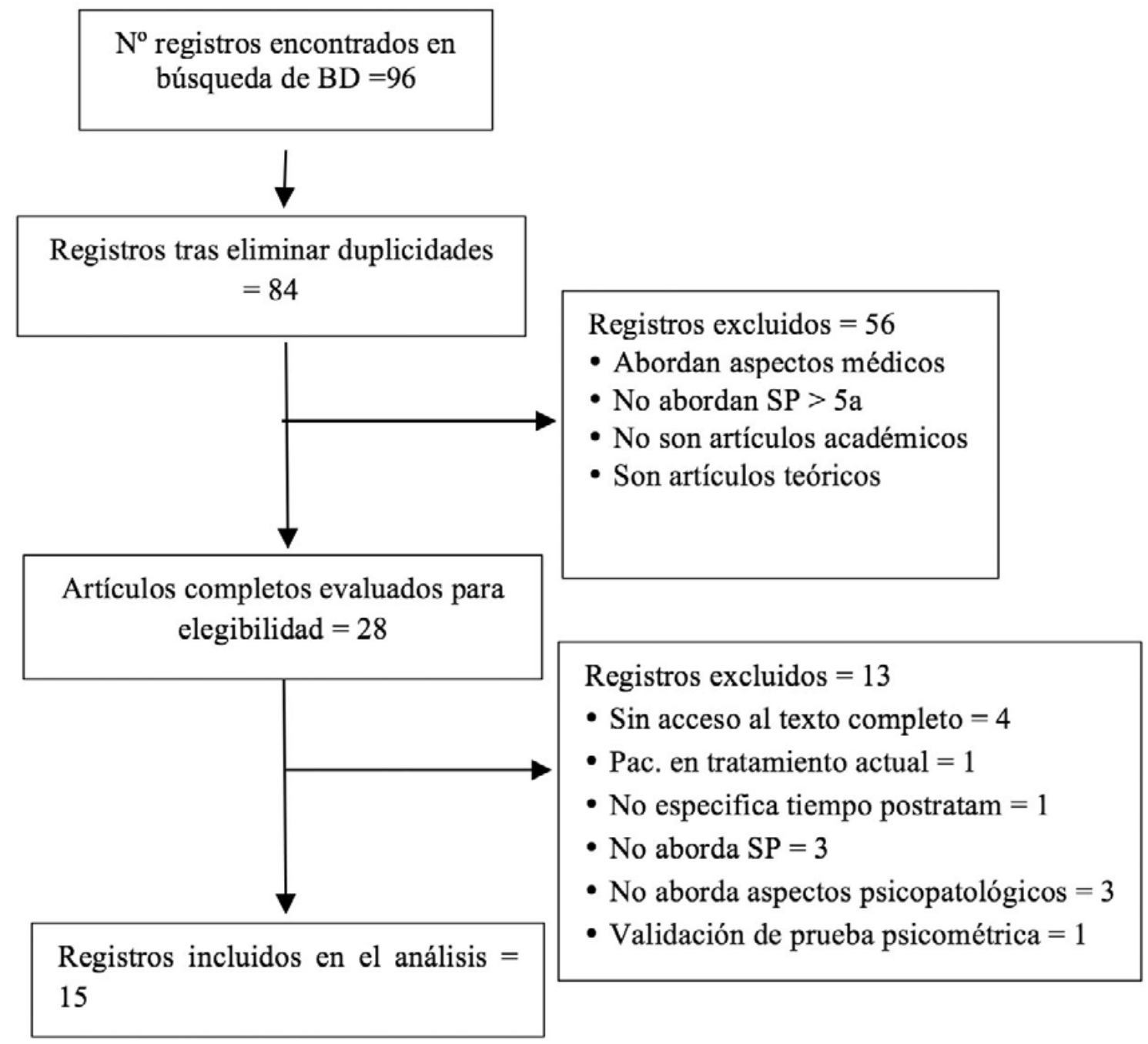

(Elaboración propia)

$\mathrm{BD}=$ Bases de datos $\mathrm{SP}=$ Supervivientes permanente 
De esta revisión exploratoria podemos extraer que la investigación con este grupo de población puede resultar complejo, ya que es una muestra que se va perdiendo en el tiempo, bien por una pérdida de interés por parte de los investigadores, bien por falta de seguimiento, por la estimación de que "curación" supone recuperación absoluta o, también, por que las mujeres pertenecientes a ese grupo poblacional no muestran deseos de ser reclutadas para este tipo de estudios. A esto se añade la discrepancia respecto al concepto de "supervivencia", diferente en función del periodo que abarca y de la entidad que lo formula.

Es evidente que a medida que transcurre el tiempo desde la enfermedad y su tratamiento, los síntomas secundarios van decreciendo. También parece evidente que la atención principal (tanto médica como psicológica) debe centrarse prioritariamente en las etapas agudas de la enfermedad. Pero también es preciso tomar en consideración la existencia de un grupo de personas cada vez mayor que requieren de una atención especializada que responda a unas necesidades específicas. La experiencia de larga supervivencia del CM supone un aspecto que va adquiriendo dimensiones lo suficientemente amplias y específicas como para detenernos en ello, ya que una nueva era de la oncología en general, y la psicooncología en particular, ha llegado para quedarse.

\section{¿Quién es superviviente y quién superviviente a largo plazo?}

Intuimos que otra razón para la escasez de investigación en el terreno que nos ocupa es la falta de consenso en la definición de supervivencia y larga supervivencia. Tradicionalmente, y desde el punto de vista biomédico, se considera que una persona es superviviente de cáncer cuando, tras haber sido diagnosticada y tratada, ha permanecido libre de la enfermedad durante 5 años, definición compartida por la SEOM. Para la OMS (Organización Mundial de la Salud) se trata de aquellos pacientes que, habiendo tenido cáncer, han seguido un tratamiento y ahora están curados, entendiendo por curación la remisión completa. En otras definiciones se conceptualiza como tal a la persona desde el mismo momento del diagnóstico de malignidad (opción que parece que se está haciendo cada vez más prevalente) y en otras se incluye a los familiares, personas cercanas y cuidadores. Como vemos, matices que establecen pequeñas pero sustanciales diferencias (Ben-Aharon et al. 2012; Wronski, 2015).

En 1985, el Dr. Fitzhugh Mullan describió las "estaciones de la supervivencia” al narrar su propia experiencia como antiguo paciente de cáncer. Para Mullan la dicotomía enfermo-curado, paciente-expaciente no abarcaba la totalidad de la experiencia. Apuntando a la presencia de la categoría "superviviente" desde el mismo momento en el que se realiza el diagnóstico (puesto que desde ese preciso instante la persona ha de replantearse su vida con nuevos parámetros), definió tres etapas en cualquier tipo de neoplasia (Mullan, 1985; Wronski, 2015):

- Supervivencia aguda: Etapa médica que incluye el momento del diagnóstico y abarca hasta la finalización del tratamiento, donde el paciente solo está pendiente de los procesos terapéuticos y sus efectos secundarios, con los miedos, temores y ansiedades que tales desafíos conllevan.

- Supervivencia extendida o extensa: Cuando el tratamiento ha finalizado y la neoplasia está en remisión, generalmente medida en meses; se produce un progresivo alejamiento de los servicios médicos, una espera vigilante en la que la persona se pregunta si los síntomas o molestias que tiene son señales de una nueva recurrencia o solo parte de la vida cotidiana, además de incertidumbre debido a las revisiones médicas sistemáticas que se siguen manteniendo y el inicio de una nueva vida aprendiendo a convivir con los efectos secundarios.

- Supervivencia permanente: La persona está libre de enfermedad y, poco a poco, va adquiriendo confianza, ya que el riesgo de recurrencia es bajo y, en teoría, ya ha dejado atrás la enfermedad y sus consecuencias. Significa que el transcurso del tiempo ya se mide en años y que la persona ya está integrada en su vida habitual; los efectos adversos del tratamiento del antiguo cáncer van disminuyendo, y los que persisten de forma crónica o permanente se incorporan al modo de vida de la persona, aunque algunos, tanto físicos como psíquicos, pueden permanecer activos. 
Teniendo en cuenta las terapias desarrolladas desde que Mullan (1985) expuso estas etapas (que siguen siendo referencia en la descripción del concepto), estas fases han tenido que reformularse, dado que la trayectoria de un sujeto que ha tenido cáncer es ahora mucho más diversa. En este sentido, Ben-Aharon et al. (2012) apuntan a la existencia de un nuevo paradigma en la descripción de las etapas (ver figura 2).

Figura 2. Etapas de la supervivencia

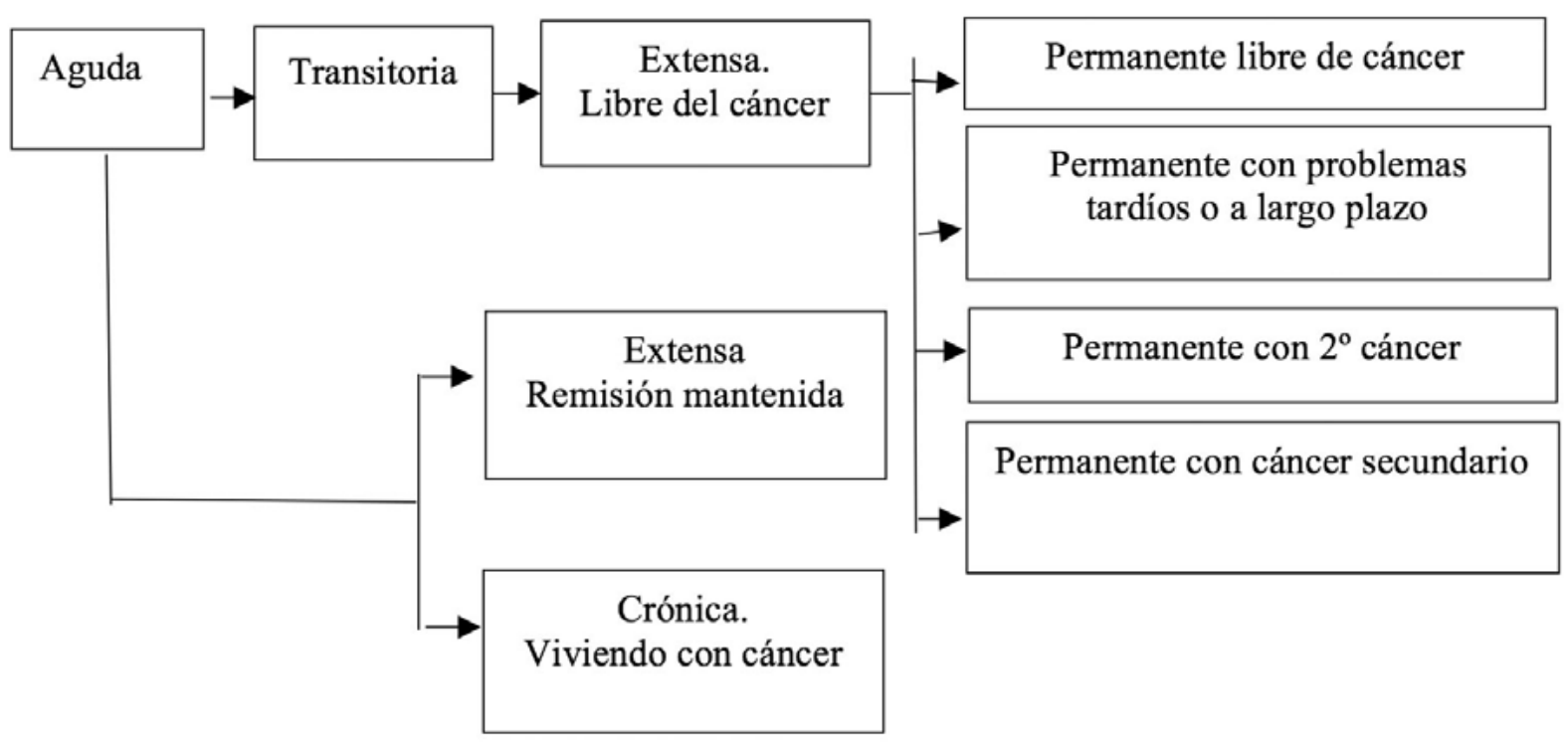

- Supervivencia aguda: Aun años después de su delimitación, los aspectos emocionales y las implicaciones de esta etapa son los mismos que los descritos por Mullan. Por tanto, como en la formulación original, recoge el periodo de diagnóstico y tratamiento.

- Transición: Supone una etapa, más o menos breve, en la que el paciente vuelve a la normalidad, aunque sería más apropiado decir una "nueva normalidad".

- Supervivencia extensa o extendida: La implicación de esta fase ha evolucionado debido a nuevas trayectorias que los pacientes pueden seguir. Tradicionalmente esta etapa supone los 5 años de observación y revisiones periódicas que la persona ha de seguir hasta que se le considere libre de enfermedad. No obstante, hoy día se deben incluir más opciones: por un lado, el mantenimiento de una medicación durante años una vez terminados los tratamientos principales (como el hormonal en CM), lo que incluye a estas personas en una "supervivencia extendida con remisión mantenida"; por otro, los "supervivientes crónicos", aquellas personas que viven con el cáncer en forma de enfermedad crónica, así como las que tienen metástasis en distintos órganos pero que, con los nuevos agentes, pueden vivir muchos años.

- Supervivencia permanente: Como señalaba Mullan (1985), incluye a aquellas personas en las cuales la neoplasia ha desaparecido hace mucho tiempo (más de 5 años) y viven libres del cáncer. No obstante, en esta fase Ben-Aharon et al. incluyen a personas "sin cáncer, pero no libres del cáncer", esto es, las personas que, libres ya de la enfermedad, siguen experimentando efectos tardíos o a largo plazo, siendo éste, el grupo objeto de nuestra atención.

Sin perder de vista las clasificaciones anteriores y para nuestro foco de interés, identificaremos superviviente con el periodo que abarca desde la finalización del tratamiento primario hasta pasados 5 años, mientras que aludimos a los supervivientes de larga evolución (o permanentes o en remisión completa) para referirnos a la persona a la que, tras haber transcurrido 5 años como mínimo, se le declara curada según los protocolos del Sistema Nacional de Salud (Ferro y Borrás, 2011). 


\section{Efectos a largo plazo}

Es habitual que, al finalizar la fase clínica y terapéutica del CM, continúen produciéndose efectos secundarios, algunos temporales, otros durante algún tiempo más, y otros que permanecen de forma más o menos crónica. No obstante, se carece de un conocimiento exacto de la incidencia de estos efectos a largo plazo, ya que la información abarca solo unos pocos años tras la finalización del tratamiento y hace referencia a CM, testicular, próstata, linfoma y cáncer infantil, todos ellos por su mayor supervivencia.

Al contemplar las quejas de los pacientes al cabo del tiempo, pueden distinguirse entre efectos tardíos y efectos a largo plazo. Los efectos tardíos incluyen aquellos que no estaban presentes o lo estaban de forma subclínica y que se manifiestan al acabar los procedimientos terapéuticos. Con efectos a largo plazo se hace referencia a síntomas colaterales que aparecen durante el tratamiento y continúan durante meses o años una vez que éste ha terminado. En referencia al CM, no existe una delimitación clara entre ambos tipos de secuelas, ya que unos investigadores clasifican los problemas cognitivos, el cansancio, el linfedema y la neuropatía periférica como efectos a largo plazo, mientras otros los incluyen en el grupo de síntomas tardíos (Ben-Aharon et al., 2012).

Sea como fuere, en la etapa de supervivencia permanente de cáncer de mama (SPCM) la paciente está libre del cáncer, lo que no implica que esté libre de problemas físicos y/o psíquicos relacionados con él. Años después del tratamiento inicial, el impacto del cáncer en la vida cotidiana de las supervivientes es escaso, aunque algunas secuelas físicas y psicológicas siguen actuando; por ende, los síntomas físicos pueden quebrantar la calidad de vida, influyendo directamente en el menoscabo del bienestar emocional. En este sentido, las SPCM refieren la permanencia de estas secuelas como algo negativo, máxime en los casos en los que nadie les informó de que podían producirse (por ejemplo, el linfedema), acudiendo para la resolución de las dudas a páginas de internet o movimientos asociacionistas. Algunos de estos síntomas a largo plazo les conducen a un nuevo carrusel médico, enfrentándose a encogimiento de hombros o a la recomendación de medicaciones no siempre eficaces y/o no siempre necesarias. Suelen ser molestias benignas que en modo alguno comprometen la supervivencia, pero que inciden en la calidad de vida y en el balance "antes-después". Sin pretender abarcar la amplia gama de repercusiones, y habida cuenta de la existencia de bibliografía al respecto, solo hacemos una breve incursión en ellos.

\section{Efectos psíquicos}

La experiencia de enfrentarse a un CM (o a cualquier otro proceso oncológico) trae consigo una serie de sentimientos que pueden oscilar desde la esperanza, la gratitud y la necesidad de protección hasta un amplio rango de emociones negativas que incluyen la ansiedad, la tristeza, la rabia, el miedo e, incluso, la culpa. Aunque todas ellas pueden ser reacciones normales en el momento del diagnóstico, en algunos casos pueden convertirse en extremas, manifestándose en forma de trastornos psicopatológicos por su intensidad y/o por su duración. La persistencia en el tiempo de secuelas físicas supone un factor de riesgo para que los sentimientos displacenteros se perpetúen, dando paso a cuadros clínicos que, aun sin conformar un síndrome completo en la mayoría de las ocasiones, hacen necesaria la atención especializada.

En las investigaciones que exploran las secuelas psíquicas a largo plazo del CM hay acuerdo en que son frecuentes los síntomas ansiosos y depresivos (Conley et al., 2016; Hernández y Cruzado, 2013) que tienden a decrecer con el paso del tiempo, aunque hay pocos trabajos que se extiendan más de dos años post-diagnóstico y que hagan un abordaje más específico respecto a síntomas concretos.

El malestar emocional o distress es un concepto excesivamente amplio y, hasta cierto punto, ambiguo que se utiliza cada vez más. Como señalan Bultz y Carlson (2005) y Hernández y Cruzado (2013), su valoración en la clínica oncológica es muy importante, considerándose como el "sexto signo vital" en la atención al cáncer (junto a la temperatura, pulso, frecuencia cardíaca y respiratoria, presión arterial y dolor), por lo que monitorizar el distrés emocional debe ser adoptado como estrategia rutinaria de la misma forma que la revisión de otros signos vitales. Definido como "experiencia emocional desagradable", este concepto abarca 
aspectos como ansiedad, depresión, malestar, disforia, ira y rabia, dando como resultado un grupo sintomatológico heterogéneo que describe de manera global el estado emocional de las pacientes de CM pero que permanece casi inexplorado en SPCM (Aaronson et al., 2014).

Aun teniendo en cuenta que el estado emocional y los síntomas de malestar cambian a medida que la paciente va pasando las etapas hasta la supervivencia a largo plazo, también existen diferentes trayectorias en la evolución de este distress en función de diferentes factores: circunstancias ambientales, apoyo social, vulnerabilidad personal, edad de la paciente o estrategias de afrontamiento que tienda a utilizar. En relación con este último aspecto, Conley et al. (2016), en su amplia revisión sobre regulación emocional en SPCM, apunta cómo mujeres con CM que suelen utilizar estrategias de regulación basadas en la desconexión (evitación conductual y cognitiva, negación, uso de sustancias y supresión) informan de mayor distress emocional, síntomas depresivos y ansiosos, menor calidad de vida y escasa satisfacción, en comparación con aquellas que adoptan una actitud de lucha y compromiso; asimismo, quienes utilizan la desconexión son más propensas a desarrollar síntomas de trastorno de estrés postraumático (TEPT). En el marco de nuestra investigación, sería conveniente conocer la evolución de este malestar en SPCM, habida cuenta de que en la mayoría de ellas este malestar disminuye sensiblemente conforme pasan los años.

Los síntomas depresivos y ansiosos son concebidos como estrategias de afrontamiento adaptativas ante la presencia de un evento evaluado como catastrófico y amenazante y que, en ocasiones, surge repentinamente. El problema aparece si el nivel es elevado pero, por su atribución de normalidad respecto al contexto, son síntomas que se pasan por alto y no son atendidos adecuadamente. A esto puede sumarse la presión de los familiares y amigos que, con todo el cariño y amor, insisten en que la persona "piense en positivo" y que "luche como una campeona", lo cual le produce a la paciente la idea de que manifestar el miedo o la tristeza es mostrar debilidad. Además, algunas temen que la tristeza afecte negativamente a su sistema inmunitario, por lo que hacen un esfuerzo ímprobo para acallar y reprimir tales emociones, lo cual puede conducir al desarrollo de un trastorno depresivo franco y a un mayor riesgo de TEPT, con síntomas que sigan activos en la etapa de larga supervivencia en forma de TEPT crónico (Leano et al., 2019).

\section{TEPT crónico}

El hecho de haber padecido una enfermedad atemorizante, con los desafíos y amenazas al bienestar que conlleva, puede considerarse dentro de la definición de evento estresante o trauma, susceptible de provocar en la persona un TEPT, si bien las mismas características de la enfermedad neoplásica confiere rasgos particulares al diagnóstico postraumático.

En el DSM-IV y el DSM-IV-TR se consideraba que los criterios diagnósticos de TEPT podrían abarcar el diagnóstico y tratamiento de enfermedades amenazantes para la vida como circunstancias elicitadoras de trauma. Sin embargo, los cambios realizados en la edición actual, el DSM-5 (APA, 2013), han supuesto una modificación sustancial en este aspecto, ya que las enfermedades orgánicas son excluidas como posibles causantes de TEPT (Cordova et al., 2017; Leano et al., 2019); de hecho, en este diagnóstico se incluyen situaciones externas, amenazas, visión o exposición a eventos atemorizantes o de riesgo, por lo que no se considerarían eventos necesariamente traumáticos las afecciones médicas potencialmente mortales o las enfermedades debilitantes (APA, 2013); según estos criterios los síntomas psíquicos en SPCM se ajustarían mejor al diagnóstico de trastorno adaptativo persistente (crónico).

Sea como fuere, son muchos los autores que abogan por la consideración de tal entidad, con la denominación de TEPT por cáncer (Cordova et al., 2017; Leano et al., 2019). Sí es cierto que encierra particularidades distintivas al TEPT que puede sufrir la población general, entre otras cosas porque los disparadores son acontecimientos interconectados más que un hecho estresante aislado, y por localizarse la amenaza en el interior del organismo y no en el exterior (Campos-Ríos, 2013; Sumalla et al., 2009). García-Torres et al. (2015) apuntan a la presencia de uno o varios síntomas del cuadro, que se agrupan en tres categorías:

1) Pensamientos intrusivos o reexperimentación en forma de pensamientos, sueños o pesadillas en relación con la enfermedad. Las secuelas físicas de los tratamientos pueden actuar como desenca- 
denantes para reexperimentar el suceso traumático (Kornblith et al, 2003), las cuales, junto a las revisiones periódicas a las que han de someterse las expacientes, suponen una reexperimentación permanente.

2) Embotamiento emocional y evitación de los recuerdos: muchas mujeres en etapa de SPCM pueden sentir cómo su terror se desencadena periódicamente ante sucesos aparentemente triviales y cotidianos: escuchar a un amigo hablar del cáncer que padece alguien, el fallecimiento de alguna mujer famosa por causa de la enfermedad, o ante las campañas mediáticas del "lazo rosa". Todo ello puede conducir a un aislamiento progresivo de su medio inmediato con el fin de evitar cualquier estímulo que le recuerde su enfermedad.

3) Hipervigilancia e hiperactivación fisiológica, donde se pueden incluir la falta de concentración o las dificultades en el inicio o mantenimiento del sueño, si bien, como afirman los autores, estos síntomas pueden constituir efectos a largo plazo del tratamiento antineoplásico, por lo que resulta difícil su adscripción.

Esta hipervigilancia es susceptible de convertirse en un síntoma a largo plazo, adquiriendo la forma de ansiedad por la salud mediante la atribución de significado potencialmente peligroso a cualquier signo corporal, adoptando la forma de preocupaciones hipocondríacas y diversas somatizaciones que, por su propia definición, ocasionan malestares físicos y trastornos funcionales sin que exista lesión orgánica que los justifiquen (Benyamini et al., 2003; Deimling et al., 2006). Por ende, esta misma atribución deletérea a cualquier signo trivial aparece marcada por el temor más generalizado en nuestra población objeto de estudio: el temor a la recurrencia, definido como el miedo o la preocupación por que el cáncer vuelva a aparecer o a evolucionar en el mismo órgano o en otra parte del cuerpo (Simard y Savall, 2009 en McGinty et al., 2016), temor que se mantiene activo aun muchos años después del carcinoma primario y con la mujer ya en la etapa de remisión total, todo lo cual puede verse incrementado si la paciente sufre determinadas secuelas permanentes del tratamiento oncogénico. Este temor, que a veces alcanza niveles clínicos, afecta al $80 \%$ de las supervivientes, incrementado en diferentes momentos como en los días previos a las revisiones, a la espera de los resultados de las pruebas, o ante cualquier síntoma físico (Martínez y Andreu, 2019). Aunque un cierto temor a la recurrencia es lógico, cuando este adquiere niveles elevados puede resultar problemático. Así, la hipervigilancia respecto al funcionamiento corporal que se desarrolla en SPCM puede constituir tanto un síntoma del TEPT crónico por cáncer como un miedo a la recurrencia en forma de nosofobia.

En 1997, Lee-Jones et al. (citados en Lebel et al., 2013) propusieron un modelo teórico sobre el miedo a la recurrencia del cáncer, según el cual este aumenta en función de varios factores: 1) la percepción de riesgo de recurrencia, lo que está influido por el estadio del cáncer primario y el tratamiento recibido; 2) estado emocional, sobre todo el grado de ansiedad, reflejado en una vigilancia aumentada respecto a sensaciones somáticas, mala interpretación de síntomas físicos benignos y conductas de evitación y búsqueda de reaseguramiento (lo cual nos conduce a los síntomas propios de la ansiedad por la salud o la nosofobia ya comentados); y 3) cuanto mayores sean las consecuencias físicas, económicas y sociales percibidas por la paciente. En este sentido, ejerce una influencia máxima el concepto de intrusión de la enfermedad concebida como la interferencia que esta supone en los roles, actividades y metas de la persona (Devins, 1994, citado en Lebel et al., 2013). Todo ello puede ocasionar peor calidad de vida, aumento de la ansiedad, pensamientos intrusivos sobre la enfermedad y, en suma, mayores síntomas de estrés postraumático. Lo que resulta evidente es que la recurrencia del proceso neoplásico adopta la forma de espada de Damocles que pende sobre las cabezas de las SPCM (y de cualquier superviviente de un proceso oncológico) y, también, sobre sus familiares, constituyendo un auténtico síndrome de Damocles (Cupit-Link et al., 2018) que puede ocasionar síntomas psíquicos.

La permanencia en el tiempo de una situación evaluada como amenazante activa el eje hipotálamo-hipofisario-adrenal (HHA) de forma crónica, con el aumento del cortisol circulante y el descenso de la capacidad defensiva del organismo al estar comprometido el sistema inmunitario. Respecto a la cuestión que nos ocupa, algunas investigaciones han mostrado un aumento del cortisol salival en mujeres SPCM, lo cual se ha asociado con altos niveles de ansiedad-rasgo, mayor malestar psicológico y peor calidad de vida (Karvinen et al., 2013). Asimismo, Starkweather et al. (2017) observaron un aumento de la proteína C-reactiva en este 
grupo de mujeres, lo cual está íntimamente relacionado con la inflamación crónica y los síntomas afectivos, si bien la investigación solo abarcó dos años post-tratamiento.

Así, y como conclusión de lo anterior, considerar el cáncer de mama como factor traumático susceptible de desencadenar un TEPT sigue siendo un asunto controvertido, aunque parece presente en un considerable porcentaje de SPCM. Por ejemplo, Chan et al. (2017) observaron que, aunque los síntomas del TEPT tendían a disminuir con el tiempo, en un $34,1 \%$ de su muestra estos persistían o, incluso, empeoraban 4 años después del tratamiento.

Con todo, se necesita clarificar los determinantes que pueden desembocar en estrés crónico para la identificación de las mujeres que están en riego alto (Lam et al., 2012). Cordova et al. (2017) y Leano et al. (2019) sugieren estar atentos en el momento del diagnóstico de la neoplasia a factores como la existencia de acontecimientos traumáticos en la vida de la paciente, a la presencia previa de TEPT u otros trastornos psicopatológicos, a la edad (más probable cuanto más joven es la paciente), y al apoyo social (más riesgo cuanto menor sea); en la fase postratamiento conviene observar si se producen síntomas disociativos respecto a la experiencia del cáncer o algún tipo de reexperimentación intrusiva.

\section{Efectos físico/funcionales con repercusión psíquica}

Si bien el tema central de nuestro trabajo apunta a las consecuencias psíquicas, creemos esencial siquiera nombrar algunas secuelas orgánicas/funcionales que van a provocar un aumento de algunos de los desórdenes antes mencionados. Obviamente, el abordaje profundo de estas cuestiones pertenece a áreas de investigación médico-oncológicas y no procede que nos detengamos más en ello.

\section{Neuropatía periférica}

El daño tisular producido por el tumor primario, los procedimientos quirúrgicos (sobre todo mastectomía) o los compuestos quimioterapéuticos pueden dar como resultado un daño en las estructuras neurales periféricas, ocasionando dolores neuropáticos que pueden tardar años en desaparecer. A esto se le conoce como síndrome doloroso post-cáncer que, para Aaronson et al. (2014), es solo una parte de un grupo de síntomas que incluye, además del síndrome doloroso, cansancio, ansiedad, depresión y trastornos del sueño.

La neuropatía periférica (adormecimiento y parestesias en manos y pies) se observa en casi todas las pacientes con carcinoma mamario que han estado en tratamiento con citotóxicos como la vincristina. Aunque la intensidad de tales molestias tiende a disminuir con el tiempo, es frecuente que persistan en algún grado durante años, constituyendo un factor estresante que sirve de recordatorio continuo del acontecimiento, disminuyendo o alterando la calidad de vida (Asociación Española del Medicamento y Productos Sanitarios [AEMPS], s. f.; Kornblith et al., 2003).

\section{Cansancio y fatiga}

La mayoría, si no todas, las pacientes de CM (y de casi todas las neoplasias) experimentan cansancio extremo durante el tratamiento. Lo que no se sabe con certeza es por qué algunas se recuperan pronto, mientras que otras permanecen durante años con esa sensación que puede convertirse en un síndrome de fatiga crónica. Obviamente, ese cansancio y falta de fuerzas tiene un profundo impacto en la calidad de vida y en el funcionamiento psíquico, sobre todo si la SPCM hace un balance entre la época pre-cáncer y la actual cuyo resultado sea la pérdida de actividades respecto a la "vida anterior". La fatiga constituye un elemento de orden multicausal, cuyo origen y evolución deberían investigarse (Aaronson et al., 2014). 


\section{Mastectomía y linfedema}

El linfedema post-mastectomía consiste en la hinchazón del brazo ipsilateral de la mama y/o ganglios linfáticos que han sido extirpados, debido a que la linfa se acumula en el tejido blando por la obstrucción de los canales linfáticos de la axila. Es un efecto secundario que ha sido descrito como una de las complicaciones más angustiosas a largo plazo de la cirugía mamaria. Esta complicación, junto a la extirpación de la mama, constituyen dos factores relevantes que inciden directamente en la imagen corporal de la mujer y en su feminidad, suponiendo una huella permanente del proceso neoplásico vivido y de las transformaciones corporales derivadas de su tratamiento.

En la revisión llevada a cabo por García-Vivar (2012) se muestra que, según las investigaciones analizadas, la imagen corporal es más favorable en supervivientes a largo plazo que se han sometido a cirugía reparadora o reconstrucción mamaria, sobre todo en pacientes jóvenes, mientras que en mujeres mayores este aspecto no encierra repercusiones significativas a largo plazo, habituándose a la utilización de prótesis y renunciando a someterse a cirugía reconstructiva.

\section{Otros efectos}

Al observar la escasez de investigaciones al respecto existentes en ese momento, Otte et al. (2010) emplearon el Pittsburgh Sleep Quality Index (PSQI) para explorar las alteraciones del sueño en mujeres SPCM. Según su estudio, los problemas en el ciclo sueño-vigilia y el insomnio parecen afectar al 65\% de ellas (media de 5,6 años desde el final del tratamiento), reflejándose también una calidad del sueño defectuosa.

La ganancia de peso es otra consecuencia del tratamiento antineoplásico, más evidente en mujeres que siguen tratamiento hormonal. Este aumento ponderal funciona como un elemento clave en el balance antes-después de muchas supervivientes, ya que observan un cuerpo transformado, modificado, al que deben acostumbrarse y que pone en cuestión su identidad (sobre todo cuando mantenían un peso normal antes del cáncer y aún más si previamente eran mujeres delgadas). De esta forma puede producirse un sentimiento de transición entre el anterior cuerpo conocido y un nuevo cuerpo extraño que se escapa a su control y que disminuye su autoconcepto (Pedersen, et al. 2016). A esto se añade cierta banalización del problema por parte del profesional, que le demanda "que haga dieta y ejercicio" sin que esa consigna resulte eficaz.

Comunes a todas las SPCM son los problemas sexuales derivados de la menopausia temprana inducida por la quimioterapia. La esterilidad, la atrofia vaginal, la amenorrea y, en suma, los síntomas menopáusicos, que pueden ser más severos que en otras mujeres sin CM, inciden de manera relevante durante muchos años después del alta. Esta cuestión es de especial preocupación para las supervivientes jóvenes por la pérdida de la capacidad reproductiva (García-Vivar, 2012), sin embargo no parece constituir fuente de distress en SPCM (Kornblit et al., 2003). Los sofocos son prevalentes y particularmente intensos en mujeres con tratamientos hormonales, como el tamoxifeno o los inhibidores de la aromatasa, lo cual supone una importante fuente de preocupación, sobre todo en situaciones de interacción social.

Como los ya comentados, otros efectos físicos a largo plazo son vividos por las SPCM como recordatorios permanentes y signos evidentes de una nueva realidad que se va plasmando con el paso del tiempo. Si bien la remisión de la neoplasia es algo por lo que se felicitan año tras año, el mayor riesgo de patología de endometrio con el tratamiento hormonal, la aceleración de problemas óseos y de articulaciones (artrosis y artritis), la cardiotoxicidad y otros problemas cardiovasculares, la disminución del rendimiento cognitivo o las alteraciones cutáneas (sobre todo relacionadas con el pelo y las uñas) implican que la persona está curada del cáncer, pero no libre de él.

\section{Superviviente como identidad}

La experiencia de haber pasado por un proceso oncológico puede traer consigo un cuestionamiento de la propia identidad, un cambio de rol y un desafío para el equilibrio mental y afectivo, lo que confiere a la 
persona que ha transitado por ese camino el apelativo de superviviente, como apuntábamos en un apartado anterior. Pese a ello, y por muy extendido y de uso común que sea, el término superviviente de cáncer (y más el de superviviente permanente) es una etiqueta con la que no todas las SPCM se sienten identificadas, ya que para algunas no significa nada, para otras es un orgullo y para otro porcentaje supone una pesada carga de la que no pueden desprenderse.

Cheung y Delfabbro (2016) realizaron una revisión de la literatura acerca de la aceptación de "superviviente de cáncer" como identidad por parte de antiguos pacientes de un proceso oncológico. Curiosamente, al examinar distintos tipos de cánceres, el 77,9\% de los individuos que superaron un cáncer de mama se identificaban como supervivientes, mientras que solo el 30,6\% de aquellos recuperados de carcinoma de próstata asumían tal apelativo.

Mediante un análisis temático, Cheung y Delfabbro, en el mismo estudio antes mencionado, categorizaron cinco grupos de personas en función de su actitud respecto a identificarse como superviviente de cáncer:

- Actitud de aceptación: Como su nombre indica, incluye a quienes representan la victoria en una batalla, a las que se sienten triunfadoras y se muestran orgullosas de ser supervivientes. Son las SPCM que personifican esa identidad creando asociaciones, recaudando fondos, encabezando campañas que periódicamente se ponen en marcha, o participando en grupos de apoyo.

- Actitud constructiva: Quienes adoptan esta actitud se preguntan qué significa exactamente ser superviviente. Por ejemplo, asumirían esta identidad si ello pudiera llevar implícito también el miedo a la recurrencia. De hecho, algunas SPCM afirman que recibir el nombre de superviviente es una forma de "tentar a la suerte".

- Actitud ambigua: No saben si quieren asumir esa identidad, si su experiencia podría acogerse a esa denominación, o hallan difícil expresar lo que significa para ellas (Williams y Jeanetta, 2015). En este sentido no sienten que su experiencia haya sido de tanta gravedad en comparación con otras formas de cáncer en las que la vida haya estado más comprometida. También pueden experimentar la sensación de haber tenido CM como si de cualquier otra enfermedad se tratara, algo que ocurrió, se trató, se curó y tras lo cual se produjo la vuelta a la vida previa, sin más cuestionamientos ni cambios.

- Actitud resistente: No les gusta ese apelativo, no quieren llevar una etiqueta, no se sienten cómodas con ella o se muestran claramente ofendidas por ello. En realidad, no desean ser miembros de ese club, ya que consideran que implica poner un foco innecesario en su enfermedad pasada, por lo que no se sienten representadas con ningún símbolo que les recuerde eternamente por lo que han pasado y cuyas consecuencias pueden seguir sufriendo.

- Actitud no destacada: O nunca han escuchado ese nombre o consideran que no ha pasado nada especial para que les llamen así. Por ejemplo, una mujer que piensa que en su vida han ocurrido situaciones mucho más determinantes que el haber sufrido un proceso oncológico ya superado.

Si bien sobrevivir al cáncer, estar curada y haber dejado atrás los tratamientos, es motivo de tranquilidad para la mayoría de las SPCM, las actitudes comentadas previamente arrojan luz sobre una cuestión que no suele reflejarse en los medios de comunicación: la de esas mujeres que no quieren asumir la identidad de superviviente como si hubieran luchado en una batalla sangrienta. La identidad positiva que se propugna como indispensable para la curación puede ser vivida como un tipo de violencia positiva en mujeres SPCM (Figueroa, 2018; Sumalla et al., 2013). La persona que se cura es catalogada como alguien excepcionalmente fuerte que se ha enfrentado a un enemigo terrible y le ha vencido, lo cual, indirectamente, le obliga a no quejarse por los síntomas tardíos que el proceso le haya ocasionado, ya que esa actitud de queja derribaría la imagen idílica de heroína que los demás (o la sociedad) le han construido.

Es posible que la sociedad no quiera ver que hay una cara B del disco. Hay una cara A, la que transmite esa valentía, la que los medios nos enseñan en las carreras populares a las que acuden millares de personas felices, sonrientes, vestidas de rosa. Es esa cara A la que obligó a las SPCM a ser buenas pacientes antes y buenas supervivientes ahora, a ser luchadoras en una batalla que no buscaron, a no pensar en las secuelas, a trivializar la negativa de los seguros médicos a suscribirles una póliza, y a participar permanentemente en el júbilo por estar vivas. 
Pero, como decíamos, hay una cara B. Esa cara B que ya en 2001 Barbara Ehrenreich, también SPCM, mostró crudamente en su artículo "Welcome to Cancerland" (citado en Sumalla et al., 2013), donde afirmaba que la positividad impuesta y opresiva, el ejército de ositos de peluche, lacitos rosas y poesías sensibleras le habían generado un malestar intenso que se sumó al malestar producido por su cáncer. De hecho, su proceso le resultó un periodo oscuro y molesto que vivió como algo injusto, le produjo rabia y que no le proporcionó ningún motivo para crecer o madurar como persona. A partir de estas declaraciones se pudo apreciar cómo esas organizaciones del lazo rosa pueden presentar una vivencia del cáncer que, para algunas expacientes, se aleja de la realidad de enfermar y de la curación, ya que, al presentar esa imagen de positividad permanente, crecimiento postraumático, sensación de alegría y ánimo expansivo, segrega a aquellas personas que no tienen esa vivencia o que, incluso, sienten reaparecer síntomas ansiosos ante la presencia permanente de los lacitos rosas, a modo de flashback postraumático. Y, por supuesto, segrega a quienes se niegan a participar de semejantes actos festivos.

De esta manera, la concepción de SPCM afín a la formulada por Mullan, aquellas supervivientes curadas de cáncer, pero no libres de sus secuelas, puede no ajustarse muy bien a la cultura del lazo rosa (Sumalla et al., 2013) donde lo predominante es la dicotomía paciente/superviviente. Obviamente esas campañas hacen sentir a un numeroso grupo de personas que no están solas, que se lucha por recaudar fondos para la investigación, que se empatiza con ellas sin ningún género de dudas, son necesarias y ayudan a la desestigmatización, pero no debemos olvidar la existencia de ese otro lado oscuro, el de aquellas SPCM que rehúyen la avalancha continua de informaciones en los medios de comunicación, las noticias (o pseudonoticias) sobre las "dietas anticáncer", a las que hacen daño las cadenas de WhatsApp que circulan una y otra vez para pedir una oración por las enfermas de cáncer o para solicitar un "me gusta" para tal o cual vídeo.

\section{Conclusión}

En las líneas anteriores hemos puesto de manifiesto la necesidad de atención especializada para las SPCM. Las dificultades de acceso a la muestra o la lógica disminución de síntomas psíquicos en etapas de curación han llevado a que la investigación en este amplio periodo se vea reducido, aunque en realidad constituye una población que crece exponencialmente conforme los avances en los tratamientos y los procedimientos de detección precoz van progresando. Esa bola de nieve de la que nos hablan Ferro y Borrás (2011) necesita atención, puesto que la mayoría de las SPCM están curadas del cáncer, pero no libres de él, tanto por los efectos a largo plazo de tipo físico como los psíquicos. La psicooncología va empezando a abordar el trabajo con este grupo particular incluyéndolo en sus actividades formativas: si bien es imprescindible la atención en el proceso diagnóstico, de tratamiento y en unidades de cuidados paliativos, la etapa de larga supervivencia constituye un reto que es preciso afrontar a la mayor brevedad. Resulta reconfortante observar cómo cada vez más unidades oncológicas van incluyendo la supervivencia permanente en sus agendas, con un equipo multidisciplinar que pueda contemplar tanto los efectos físicos tardíos del proceso antineoplásico como los psicológicos; estos últimos pueden conformar síndromes subclínicos o claramente psicopatológicos, adoptando distintas formas, como el TEPT crónico por cáncer.

El presente trabajo presenta limitaciones inherentes a un artículo de reflexión teórica. El abordaje de una revisión sistemática de los artículos hallados en la búsqueda, el reclutamiento de una muestra de SPCM para la constatación (o no) de las secuelas psicológicas que formulamos como hipótesis, la comprobación de la existencia de un TEP crónico (clínico o subclínico) en parte de esta población, o la exploración de síntomas de ansiedad por la salud o trastornos somatomorfos más allá del miedo a la recurrencia, quedarían como futuras investigaciones que podrían llevarse a cabo. Pero queden estas líneas como una llamada de atención sobre algo que requiere mayor investigación, ya que las supervivientes a largo plazo ( $>5$ años después del tratamiento) siguen necesitando apoyo del campo de la psicooncología. 


\section{Referencias}

Aaronson, N. K., Mattioli, V., Minton, O., Weis, J., Johansen, C., Dalton, S. O., Verdonck-de Leeuw, I. M., Stein, K. D., Alfano, C. M., Mehnert, A., de Boer, A. y van de Poll-Franse, L. K. (2014). Beyond treatment - Psychosocial and behavioural issues in cancer survivorship research and practice. European Journal of Cancer Supplements 12(1), 54-64. http://doi.org/10.1016/j.ejcsup.2014.03.005

Asociación Española Contra el Cáncer (AECC). (2019). Supervivencia cáncer de mama. http://www.aecc.es/ es/todo-sobre-cancer/tipos-cancer/cancer-mama/mas-informacion/evolucion-cancer-mama

Agencia Española de Medicamentos y Productos Sanitarios (AEMPS). (s. f.). http://www.aemps.gob.es/

Álvarez-Salvago, F., Galiano-Castillo, N., Arroyo-Morales, M., Cruz-Fernández, M., Lozano-Lozano, M. y Cantarero-Villanueva, I. (2018). Health status among long-term breast cancer survivors suffering from higher levels of fatigue: a cross-sectional study. Supportive Care in Cancer, 26(10), 3649-3658. https://doi. org/10.1007/s00520-018-4240-z

American Psychiatric Association (APA). (2013). Manual Diagnóstico y Estadístico de los Trastornos Mentales (5 $5^{\mathrm{a}}$ ed., DSM-5). Médica Panamericana.

Ben-Aharon, I., Miller, K. y Haines, L. (2012). Seasons of survival. Oncology Practice Management, 2(1). http://oncpracticemanagement.com/issues/2012/february-2012-vol-2-no-1/269-seasons-of-survival/

Benyamini, Y., McClain, C. S., Leventhal, E. A. y Leventhal, H. (2003). Living with the worry of cancer: Health perceptions and behaviors of elderly people with self, vicarious or no history of cancer. Psycho-Oncology, 12(2), 161-172. https://doi.org/10.1002/pon.637

Bultz, B. D. y Carlson, L. E. (2005). Emotional distress: the sixth vital sign in cancer care. Journal of Clinical Oncology, 23(26), 6440-6441. https://doi.org/10.1200/JCO.2005.02.3259

Campos-Ríos, M. M. (2013). Creciendo en la adversidad. Una revisión del proceso de adaptación al diagnóstico de cáncer y el crecimiento postraumático. Escritos de Psicología 6(1), 6-13. https://doi.org/10.5231/psy. writ.2012.2109

Chan, C. M. H., Ng, C. G., Taib, N. A., Wee, L. H., Krupat, E. y Meyer, F. (2017). Course and predictors of post-traumatic stress disorder in a cohort of psychologically distressed patients with cancer: a 4-year follow-up study. Cancer, 124(2), 406-416. https://doi.org/10.1002/cncr.30980

Cheung, S. Y. y Delfabbro, P. (2016). Are you a cancer survivor? A review on cancer identity. Journal of Cancer Survivorship, 10, 759-771. https://doi.org/10.1007/s11764-016-0521-z

Conley, C. C., Bishop, B. T. y Andersen, B. L. (2016). Emotions and Emotion Regulation in Breast Cancer Survivorship. Healthcare, 4(56). https://doi.org/10.3390/healthcare4030056

Cordova, M. J., Riba, M. B. y Spiegel, D. (2017). Post-traumatic stress disorder and cancer. Lancet Psychiatry, 4(4), 330-338. https://doi.org/10.1016/S2215-0366(17)30014-7

Cupit-Link, M., Syrjala, K. L. y Hashmi, S. K. (2018). Damocles'syndrome revisited: Update on the fear of cancer recurrence in the complex world of today's treatments and survivorship. Hematology/Oncology and Stem Cell Therapy, 11(3), 129-134. https://doi.org/10.1016/j.hemonc.2018.01.005

Deimling, G. T., Bowman, K. F., Sterns, S. Wagner, L. J. y Kahana, B. (2006). Cancer-related health worries and psychological distress among older adult, long-term cancer survivors. Psycho-Oncology, 15(4), 306320. https://doi.org/10.1002/pon.955

Ferro, T. y Borràs, J. M. (2011). Una bola de nieve está creciendo en los servicios sanitarios: los pacientes supervivientes de cáncer. Gaceta Sanitaria, 25(3), 240-245.

Figueroa, M. R. (2018). La identidad positiva como violencia simbólica en mujeres con cáncer. Géneros. Revista de Investigación y Divulgación sobre los Estudios de Género, 22(2), 133-156.

Galindo, O., Rojas, E., Benjet, C., Meneses, A., Aguilar, J. L. y Alvarado, S. (2014). Efectos de las intervenciones psicológicas en supervivientes de cáncer: una revisión. Psicooncología, 11(2-3), 233-241. https://doi. org/10.5209/rev PSIC.2014.v11.n2-3.47385

García-Torres, F., Alós, F. J. y Pérez-Dueñas, C. (2015). El trastorno por estrés postraumático en los supervivientes al cáncer: una revisión de los tratamientos psicológicos disponibles. Psicooncología, 12(2-3), 293301. https://doi.org/10.5209/rev PSIC.2015.v12.n2-3.51010 
García-Vivar, C. (2012). Impacto psicosocial del cáncer de mama en la etapa de larga supervivencia: propuesta de un plan de cuidados integral para supervivientes. Atención Primaria, 44(5), 288-292. https://doi. org/10.1016/j.aprim.2011.07.011

García-Vivar, C. y McQueen, A. (2005). Informational and emotional needs of long-term survivors of breast cancer. Journal of Advanced Nursing, 51(5), 520-528.

Harrington, C. B., Hansen, J. A., Moskowitz, M., Todd, B. L. y Feuerstein, M. (2010). It's not over when it's over: Long-term symptoms in cancer survivors. A systematic review. International Journal of Psychiatry in Medicine, 40(2), 163-181. https://doi.org/10.2190/PM.40.2.c

Hernández, M. y Cruzado, J. A. (2013). La atención psicológica a pacientes con cáncer: de la evaluación al tratamiento. Clínica y Salud, 24(1), 1-9. https://doi.org/10.5093/cl2013a1

Hewitt, M., Greenfield, S. y Stovall, E. (2006). From cancer patient to cancer survivor: Lost in transition. The National Academies Press.

Karvinen, K. H., Murray, N. P., Arastu, H. y Allison, R. R. (2013). Stress reactivity, health behaviors and compliance to medical care in breast cancer survivors. Oncology Nursing Forum, 40(2), 149-156.

Kornblith, A. B., Herndon, J. E., Weiss, R. B., Zhang, C., Zuckerman, E. L., Rosenberg, S., Mertz, M., Payne, D., Massie, M. J., Holland, J. F., Wingate, P., Norton, L. y Holland, J. C. (2003). Long-Term Adjustment of Survivors of Early-Stage Breast Carcinoma, 20 Years after Adjuvant Chemotherapy. American Cancer Society 98(4), 679-689. https://doi.org/10.1002/cncr.11531

Lam, W. W. T., Shing, Y. T., Bonnano, G. A., Mancini, A. D. y Fielding, R. (2012). Distress trajectories at the first-year diagnosis of breast cancer in relation to 6 years survivorship. Psycho-Oncology 21(1), 90-99. https://doi.org/10.1002/pon.1876

Leano, A., Korman, M. B., Goldberg, L. y Ellis, J. (2019). Are we missing PTSD in our patients with cancer? Part I. Canadian Oncology Nursing Journal 29(2), 141-146.

Lebel, S., Beattie, S., Arès, I. y Bielajew, C. (2013). Yong and worried: Age and fear of recurrence in Breast Cancer Survivors. Health Psychology 32(6), 695-705. https://doi.org/10.1037/a0030186

Martínez, P. y Andreu, Y. (2019). Supervivencia en cáncer: la necesidad de una atención integral y la importancia de asumir un rol activo. Papeles del Psicólogo 40(1), 1-7. https://doi.org/10.23923/pap.psicol2019.2885

McGinty, H. L., Small, B. J., Laronga, C. y Jacobsen, P. B. (2016). Predictors and patterns of fear of cancer recurrence in breast cancer survivors. Health Psychology 35(1), 1-9. https://doi.org/10.1037/hea0000238

Mullan, F. (1985). Seasons of survival: reflections of a physician with cancer. The New England Journal of Medicine, 313, 270-273. https://doi.org/10.1056/NEJM198507253130421

Otte, J. L., Carpenter, J. S., Russell, K. M., Bigatti, S. y Champion, V. L. (2010). Prevalence, severity and correlates of sleep-wake disturbances in long term breast cancer survivors. Journal of Pain and Symptom Management, 39(3), 535-547.

Padilla-Ruiz, M., Ruiz-Román, C., Pérez-Ruiz, E., Rueda, A., Redondo, M. y Rivas-Ruiz, F. (2019). Clinical and sociodemographic factors that may influence the resilience of women surviving breast cancer: cross-sectional study. Supportive Care in Cancer, 27, 1279-1286. https://doi.org/10.1007/s00520-018-4612-4

Pedersen, B., Groenkjaer, M., Falkmer, U., Mark, E. y Delmar, Ch. (2016). 'The ambiguous transforming body' - A phenomenological study of the meaning of weight changes among women treated for breast cancer. International Journal of Nursing Studies, 55, 15-25.

Roales-Nieto, J. G. (2016). En manos del sistema: cómo sobrevivir a la supervivencia. Dykinson.

Sociedad Española de Oncología Médica (SEOM). (2012). Manifiesto SEOM sobre la Atención a los Largos Supervivientes de Cáncer. https://seom.org/publicaciones/publicaciones-seom/largos-supervivientes/103911-manifiesto-seom-sobre-la-atencion-a-los-largos-supervivientes-de-cancer

Sociedad Española de Oncología Médica (SEOM). (2017). Recomendaciones para el seguimiento de las mujeres supervivientes de Cáncer de Mama. https://seom.org/publicaciones/publicaciones-seom/largos-supervivientes

Sociedad Española de Oncología Médica (SEOM). (2018). Plan integral de atención a los largos supervivientes de cáncer. https://www.seom.org/seomcms/images/stories/recursos/PLAN INTEGRAL LARGO SUPERVIVIENTE.pdf 
Starkweather, A., Kelly, D. L., Thacker, L., Wright, M. L., Jackson-Cook, C. K. y Lyon, D. E. (2017). Relationships among psychoneurological symptoms and levels of C-reactive protein over 2 years in women with early-stage breast cancer. Support Care Cancer, 25(1), 167-176.

Sumalla, E. C., Ochoa, C., Blanco, I. (2009). Posttraumatic growth in cancer: reality or illusion? Clinical Psychology Review 29, 24-33. https://doi.org/10.1016/j.cpr.2008.09.006

Sumalla, E. C., Castejón, V., Ochoa, C. y Blanco, I. (2013). ¿Por qué las mujeres con cáncer de mama deben estar guapas y los hombres con cáncer de próstata pueden ir sin afeitar? Oncología, disidencia y cultura hegemónica. Psicooncología, 10(1), 7-56. https://doi.org/10.5209/rev PSIC.2013.v10.41946

Williams, F. y Jeanetta, S. C. (2015). Lived experiences of breast cancer survivors after diagnosis, treatment and beyond: qualitative study. Health Expectations, 19, 631-642. https://doi.org/10.1111/hex.12372

Wronski, A. L. (2015). Defining cancer survivor and cancer survivorship: the who, what and when. Psicooncología, 12(1), 7-18. https://doi.org/10.5209/rev PSIC.2015.v12.n1.48900

Artículo recibido: 26/07/2020

Artículo aceptado: 28/09/2021 